\title{
Enhanced reaction kinetics in biological cells
}

\author{
C. LOVERDO, O. BÉNICHOU, M. MOREAU AND R. VOITURIEZ* \\ Laboratoire de Physique Théorique de la Matière Condensée, Université Pierre et Marie Curie, 4 Place Jussieu, 75252 Paris cedex 05, France \\ *e-mail: voiturie@Iptmc.jussieu.fr
}

The cell cytoskeleton is a striking example of an 'active' medium driven out-of-equilibrium by ATP hydrolysis ${ }^{1}$. Such activity has been shown to have a spectacular impact on the mechanical and rheological properties of the cellular medium ${ }^{2-10}$, as well as on its transport properties ${ }^{11-14}$ : a generic tracer particle freely diffuses as in a standard equilibrium medium, but also intermittently binds with random interaction times to motor proteins, which perform active ballistic excursions along cytoskeletal filaments. Here, we propose an analytical model of transport-limited reactions in active media, and show quantitatively how active transport can enhance reactivity for large enough tracers such as vesicles. We derive analytically the average interaction time with motor proteins that optimizes the reaction rate, and reveal remarkable universal features of the optimal configuration. We discuss why active transport may be beneficial in various biological examples: cell cytoskeleton, membranes and lamellipodia, and tubular structures such as axons ${ }^{1}$.

Various motor proteins such as kinesins or myosins are able to convert the chemical fuel provided by ATP into mechanical work by interacting with the semiflexible oriented filaments (mainly F-actin and microtubules) of the cytoskeleton ${ }^{1}$. As many molecules or larger cellular organelles such as vesicles, lysosomes or mitochondria, hereafter referred to as tracer particles, can randomly bind and unbind to motors, the overall transport of a tracer in the cell can be described as alternating phases of standard diffusive transport and phases of active directed transport powered by motor proteins ${ }^{1,15,16}$. Active transport in cells has been extensively studied both experimentally, for instance by single-particle tracking methods ${ }^{11,12}$, and theoretically by evaluating the mean displacement of a tracer ${ }^{13,17}$, or stationary concentration profiles ${ }^{14}$.

On the other hand, most cell functions are regulated by coordinated chemical reactions that involve low concentrations of reactants (such as ribosomes or vesicles carrying targeted proteins), and are therefore limited by transport. However, up to now a general quantitative analysis of the impact of active transport on reaction kinetics in cells, and more generally in generic active media, is still missing, even though a few specific examples have been tackled ${ }^{18}$. Here, we propose an analytical model that enables us to determine for the first time the kinetic constant of transportlimited reactions in active media.

The model relies on the idea of intermittent dynamics introduced in the context of search processes ${ }^{19-27}$. We consider a tracer particle evolving in a $d$-dimensional space (in practice $d=1,2,3$ ) that exhibits thermal diffusion phases of diffusion coefficient $D$ (denoted phases 1 ), randomly interrupted by ballistic excursions bound to motors (referred to as phases 2) of constant velocity $v$ and direction pointing in the solid angle $\omega_{\mathrm{v}}$ (Fig. 1a). The distribution of the filaments' orientation is denoted by $\rho\left(\omega_{\mathbf{v}}\right)$, and will be taken as either disordered or polarized (Fig. 1a), which schematically reproduces the different states of the cytoskeleton $^{1}$. The random duration of each phase $i$ is assumed to be exponentially distributed with mean $\tau_{i}$. The tracer $T$ can react with reactants $R$ (supposed immobile) only during free-diffusion phases 1 , as $T$ is assumed to be inactive when bound to motors, which is realized for instance when the reactants are membrane proteins (Fig. 1b,c). Reaction occurs with a finite probability per unit of time $k$ when the tracer-reactant distance is smaller than a given reaction radius $a$. In what follows, we explicitly determine the kinetic constant $K$ of the reaction $T+R \rightarrow R$.

We now present the basic equations in the case of a reactant centred in a spherical domain of radius $b$ with a reflecting boundary. This geometry both mimics the relevant situation of a single target and provides a mean-field approximation of the general case of randomly located reactants with concentration $c=a^{d} / b^{d}$, where $b$ is the typical distance between reactants. We start from a mean-field approximation of the first-order reaction constant ${ }^{28}$ and write $K=1 /\langle t\rangle$, where the key quantity of our approach is the reaction time $\langle t\rangle$, which is defined as the mean first-passage time ${ }^{29,30}$ (MFPT) of the tracer at a reactant position uniformly averaged over its initial position. For the active intermittent dynamics defined above, the MFPT of the tracer at a reactant position satisfies the following backward equation ${ }^{29}$ :

$$
\left\{\begin{array}{l}
D \Delta_{\mathrm{r}} t_{1}+\frac{1}{\tau_{1}} \int\left(t_{2}-t_{1}\right) \rho\left(\omega_{\mathbf{v}}\right) \mathrm{d} \omega_{\mathbf{v}}-k \mathrm{I}_{a}(\mathbf{r}) t_{1}=-1 \\
\mathbf{v} \cdot \nabla_{\mathbf{r}} t_{2}-\frac{1}{\tau_{2}}\left(t_{2}-t_{1}\right)=-1,
\end{array}\right.
$$

where $t_{1}$ is the MFPT starting in phase 1 at position $\mathbf{r}$, and $t_{2}$ is the MFPT starting in phase 2 at position $\mathbf{r}$ with velocity $\mathbf{v} . \mathrm{I}_{a}$ is the indicator function of the ball of radius $a$. As these equations (1) are of integro-differential type, standard methods of resolution are not available for a general distribution $\rho$.

However, in the case of a disordered distribution of filaments ( $\rho\left(\omega_{\mathrm{v}}\right)=1 / \Omega_{d}$, where $\Omega_{d}$ is the solid angle of the $d$-dimensional sphere), we can use a generalized version of the decoupling approximation introduced in ref. 23 to obtain a very good approximate solution of equations (1), as described in the Methods section. Here, we present simplified expressions of the resulting kinetic constant by taking alternatively the limit $k \rightarrow \infty$, which corresponds to the ideal case of perfect reaction, and the limit $D \rightarrow 0$, which enables us to isolate the $k$ dependence.

First we discuss the $d=3$ disordered case (Fig. 1a), which provides a general description of the actin cytoskeleton of a cell in nonpolarized conditions, or of a generic in vitro active solution. An analytical form of the reaction rate $K_{3 d}$ is given in the Methods 

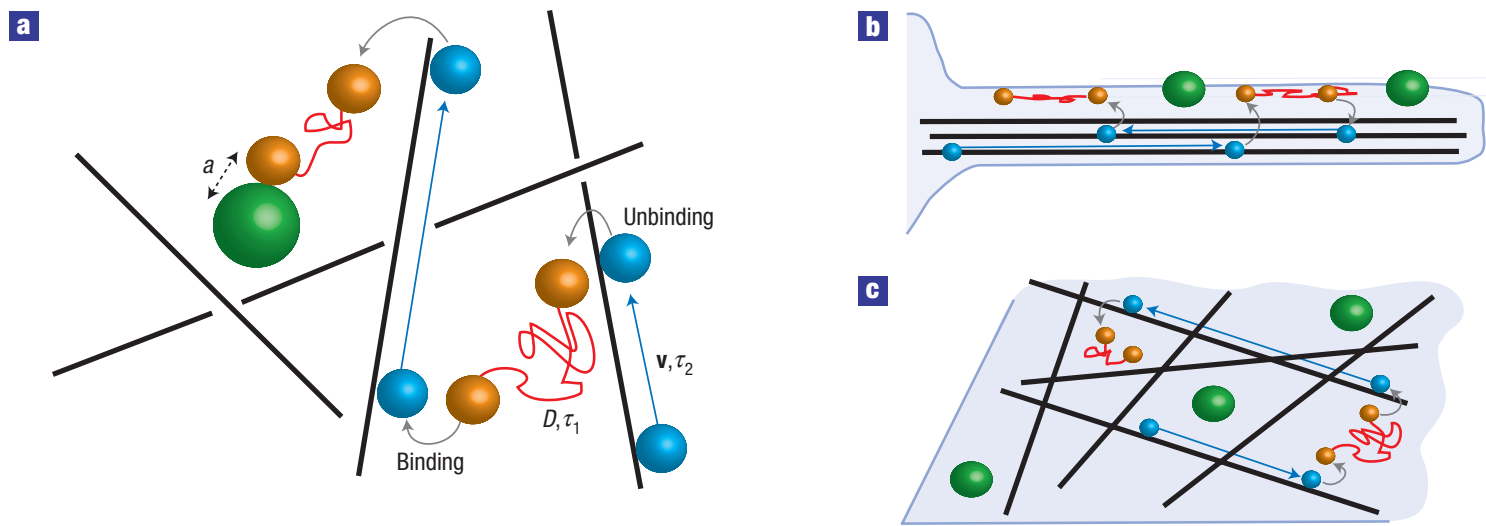

Figure 1 Model of reaction kinetics in active media, and examples of low-dimensional structures in biological cells. a, The reactant alternates thermal diffusion phases (regime 1 in red) of mean duration $\tau_{1}$ and diffusion coefficient $D$, and ballistic phases of velocity $\mathbf{v}$ powered by molecular motors (regime 2 in blue) of mean duration $\tau_{2}$. Here, the cytoskeletal filaments (in black) are in a disordered state. The polarized nematic state would correspond to parallel filaments, and is equivalent in a first approximation to a one-dimensional situation (see $\mathbf{b}$ ) with the same concentration $c_{1 d}=c_{3 d}=a_{3 d}^{3} / b_{3 d}^{3}$ and an effective reaction radius $a_{1 d}=a_{3 d} c_{3 d}^{2 / 3}$. Molecular motors are not represented. $\mathbf{b}$, Tubular structures in cells such as axons and dendrites $(d=1)$. c, Planar structures such as membranes and lamellipodia $(d=2)$.

section, and is plotted in Fig. 2a,b. Strikingly, $K_{3 d}$ can be maximized (Fig. 2a,b) as soon as the reaction radius exceeds a threshold $a_{\mathrm{c}} \simeq D / v$ for the following value of the mean interaction time with motors:

$$
\tau_{2,3 d}^{\mathrm{opt}}=\frac{\sqrt{3} a}{v x_{0}} \simeq 1.078 \frac{a}{v}
$$

where $x_{0}$ is the solution of $2 \tanh (x)-2 x+x \tanh (x)^{2}=0$. The $\tau_{1}$ dependence is very weak, but we can roughly estimate the optimal value by $\tau_{1,3 d}^{\mathrm{opt}} \simeq 6 D / v^{2}$. This in turn gives the maximal reaction rate

$$
K_{3 d}^{m} \simeq \frac{c v}{a} \frac{\sqrt{3}\left(x_{0}-\tanh \left(x_{0}\right)\right)}{x_{0}^{2}},
$$

so that the gain with respect to the reaction rate $K_{3 d}^{p}$ in a passive medium is $G_{3 d}=K_{3 d}^{m} / K_{3 d}^{p} \simeq \operatorname{Cav} / D$ with $C \simeq 0.26$.

Several comments are in order. (1) $\tau_{2,3 d}^{\mathrm{opt}}$ neither depends on $D$, nor on the reactant concentration. A similar analysis for $k$ finite (in the $D \rightarrow 0$ limit) shows that this optimal value does not depend on $k$ either, which proves that the optimal mean interaction time with motors is widely independent of the parameters characterizing the diffusion phase 1. (2) The value $a_{\text {c }}$ should be discussed. In standard cellular conditions, $D$ ranges from $\simeq 10^{-2} \mu \mathrm{m}^{2} \mathrm{~s}^{-1}$ for vesicles to $\simeq 10 \mu \mathrm{m}^{2} \mathrm{~s}^{-1}$ for small proteins, whereas the typical velocity of a motor protein is $v \simeq 1 \mu \mathrm{m} \mathrm{s}^{-1}$, a value that is widely independent of the size of the cargo ${ }^{1}$. This gives a critical reaction radius $a_{\mathrm{c}}$ ranging from $\simeq 10 \mathrm{~nm}$ for vesicles, which is smaller than any cellular organelle, to $\simeq 10 \mu \mathrm{m}$ for single molecules, which is comparable to the whole cell dimension. Hence, this shows that in such a three-dimensional disordered case, active transport can optimize reactivity for sufficiently large tracers such as vesicles, as motor-mediated motion permits a fast relocation to unexplored regions, whereas it is inefficient for standard molecular reaction kinetics, mainly because at the cell scale molecular free diffusion is faster than motor-mediated motion. This could help justify that many molecular species in cells are transported in vesicles. Interestingly, in standard cellular conditions $\tau_{2,3 d}^{\mathrm{opt}}$ is of the order of $0.1 \mathrm{~s}$ for a typical reaction radius of the order of $0.1 \mu \mathrm{m}$. This value is compatible with experimental observations ${ }^{1}$, and suggests that cellular transport is close to optimum. (3) The typical gain for a vesicle of reaction radius $a \gtrsim 0.1 \mu \mathrm{m}$ in standard cellular conditions is $G_{3 d} \gtrsim 2.5$ (Fig. 2a,b) and can reach $G_{3 d} \gtrsim 10$ for faster types of molecular motor such as myosins ( $v \simeq 4 \mu \mathrm{m} \mathrm{s}^{-1}$, see refs 1,11$)$, independently of the reactant concentration $c$. As we shall show below, the gain will be significantly higher in lower-dimensional structures such as axons.

We now come to the $d=2$ disordered case (Fig. 1c). Striking examples in cells are given by the cytoplasmic membrane, which is closely coupled to the network of cortical actin filaments, or the lamellipodium of adhering cells ${ }^{1}$. In many cases, the orientation of filaments can be assumed to be random. This problem then exactly maps the search problem studied in ref. 23, where the reaction time was calculated. This enables us to show that as for $d=3$, the reaction rate $K_{2 d}$ can be optimized in the regime $D / v \ll a \ll b$. Remarkably, the optimal interaction time $\tau_{2,2 d}^{\mathrm{opt}}$ takes the same value in the two limits $k \rightarrow \infty$ and $D \rightarrow 0$ :

$$
\tau_{2,2 d}^{\mathrm{opt}} \simeq \frac{a}{v}\left(\ln \left(1 / c^{1 / 2}\right)-1 / 2\right)^{1 / 2},
$$

which indicates that again $\tau_{2,2 d}^{\text {opt }}$ does not depend on the parameters of the thermal diffusion phase, neither through $D$ nor $k$. In the limit $k \rightarrow \infty$, we have $\tau_{1,2 d}^{\mathrm{opt}}=\left(D / 2 v^{2}\right)\left(\ln ^{2}\left(1 / c^{1 / 2}\right) /\left(2 \ln \left(1 / c^{1 / 2}\right)-1\right)\right)$, and the maximal reaction rate can then be obtained:

$$
K_{2 d}^{m} \simeq \frac{c^{1 / 2} v}{2 a \sqrt{\ln \left(1 / c^{1 / 2}\right)}} .
$$

Comparing this expression to the case of passive transport yields a gain $G_{2 d}=K_{2 d}^{m} / K_{2 d}^{p} \simeq a v \sqrt{\ln \left(1 / c^{1 / 2}\right)} /(4 D)$. As in the $d=3$ case, this proves that active transport enhances reactivity for large enough tracers (with a critical reaction radius $a_{\mathrm{c}} \simeq D / v$ of the same order as in the $d=3$ case) such as vesicles. However, here the gain $G_{2 d}$ depends on the reactant concentration $c$, and can be more significant: with the same values of $D, v$ and $a$ as given above for a vesicle in standard cellular conditions, and for low concentrations of reactants (such as specific membrane receptors) with a typical distance between reactants $b \gtrsim 10 \mu \mathrm{m}$, the typical gain is $G_{2 d} \gtrsim 8$, and reaches 10 for single reactants (such as some signalling molecules).

The case of nematic order of the cytoskeletal filaments, which depicts for instance the situation of a polarized cell ${ }^{1}$, can be shown 
a

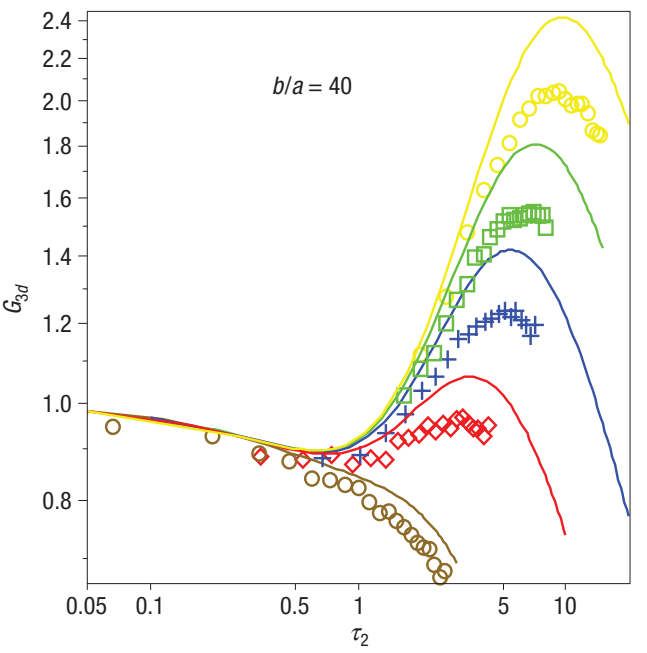

G

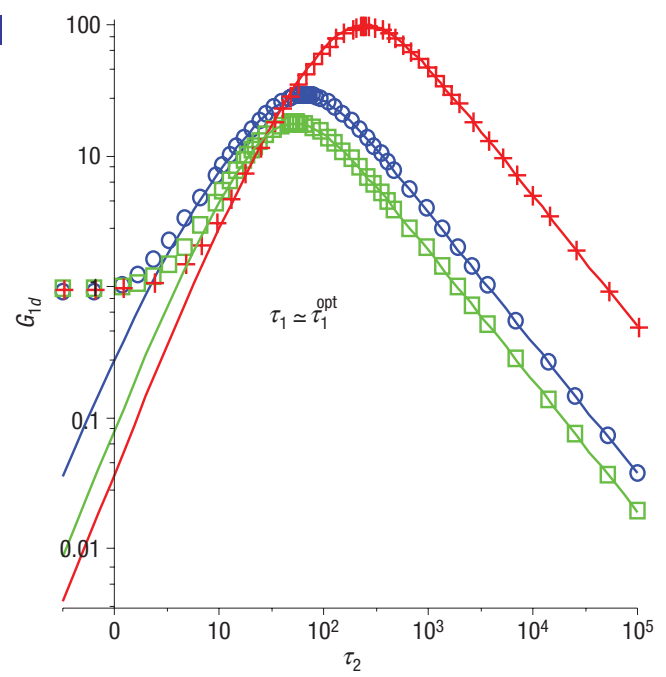

b

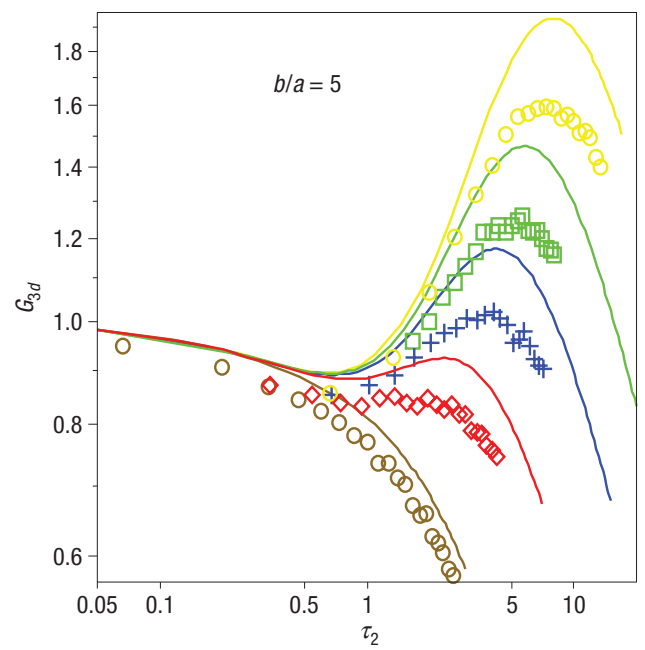

d

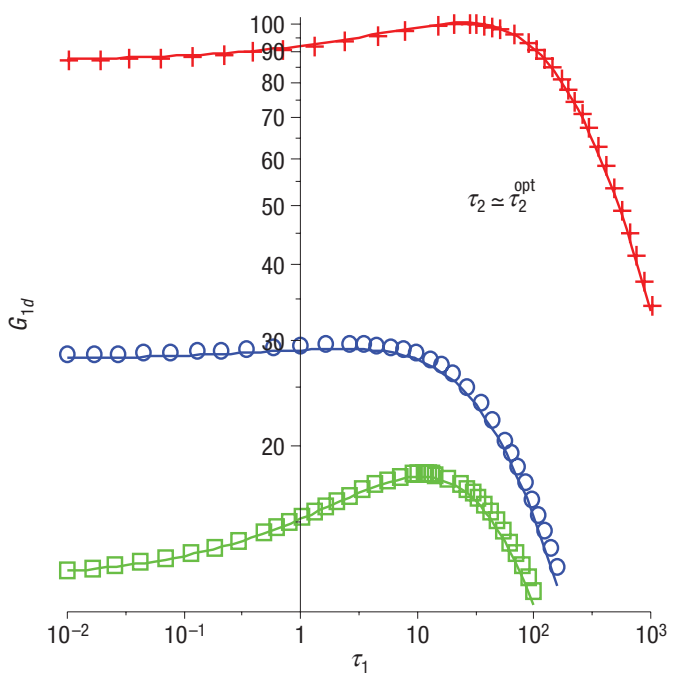

Figure 2 Optimization of the reaction rate. a,b, Gain of reactivity due to active transport $G_{3 d}$ for $d=3$ as a function of $\tau_{2}$ for different values of the ratio $b / a$ (logarithmic scale). The analytical form obtained in the Methods section (lines) is plotted against numerical simulations (symbols) for the following values of the parameters (arbitrary units): $a=1.5$ (brown), $a=4.5$ (red), $a=7.5$ (blue), $a=10.5$ (green), $a=15$ (yellow), with $\tau_{1}=6, v=1$ and $D=1 . K_{3 d}$ presents a maximum only for $a>a_{\mathrm{c}} \simeq 4$. Standard cellular conditions (as discussed in the text) correspond to green and yellow curves for $b / a=40$. c,d, Gain of reactivity due to active transport $G_{1 d}$ for $d=1$ as a function of $\tau_{2}$ (c) and $\tau_{1}$ (d) (logarithmic scale). The analytical form obtained in the Methods section (lines) is plotted against the exact solution (symbols), for the following values of the parameters (arbitrary units): $D=1$ and $v=1$ for all curves and $a=10, b=10^{4}$ (red), $a=10, b=10^{3}$ (blue) and $a=2.5, b=10^{3}$ (green). Standard cellular conditions (as discussed in the text) correspond to blue and red curves.

to be equivalent in a first approximation to the one-dimensional case, which is exactly solvable (Fig. 1a,b). The $d=1$ case is also important on its own in cell biology, as many one-dimensional active structures such as axons, dendrites or stress fibres ${ }^{1}$ are present in living cells. As an illustration, we take the example of an axon, filled with parallel microtubules pointing their plus end in a direction e. We consider a tracer particle interacting with both kinesins (' + ' end directed motors, of average velocity ve) and dyneins ('-' end directed motors, of average velocity $-v \mathbf{e}$ ) with the same characteristic interaction time $\tau_{2}$ (see Fig. 1b). For this type of tracer, the MFPT satisfies equations (1) with an effective nematic distribution of filaments $\rho\left(\omega_{\mathbf{v}}\right)=(1 / 2)(\delta(\mathbf{v}-\mathbf{e})+\delta(\mathbf{v}+\mathbf{e}))$. The reaction rate $K_{1 d}$ is obtained exactly in this case (see the Methods section), and is maximized in the regime $D / v \ll a \ll b$ for the following values of the characteristic times (see Fig. 2c,d)

$$
\tau_{1,1 d}^{\mathrm{opt}}=\frac{1}{48} \frac{D}{v^{2} c}, \quad \tau_{2,1 d}^{\mathrm{opt}}=\frac{1}{\sqrt{3}} \frac{a}{v c^{1 / 2}},
$$

for $k \rightarrow \infty$. The maximal reaction rate $K_{1 d}^{m}$ is then given by

$$
K_{1 d}^{m} \simeq \frac{\sqrt{3} v c^{3 / 2}}{2 a}
$$

and the gain is $G_{1 d}=K_{1 d}^{m} / K_{1 d}^{p} \simeq a v /\left(2 \sqrt{3} D c^{1 / 2}\right)$, which proves that active transport can optimize reactivity as in higher dimensions. Interestingly, the $c$ dependence of the gain is much more important than for $d=2,3$, which shows that the efficiency of active transport is strongly enhanced in one-dimensional or nematic structures at low concentration. Indeed, with the same values of $D, v$ and 
$a$ as given above for a vesicle in standard cellular conditions, and for a typical distance between reactants $b \gtrsim 100 \mu \mathrm{m}$ (such as low concentrations of axonal receptors), we obtain a typical gain $G_{1 d} \gtrsim 100$ (see Fig. $2 c, d$ ). In the limit of finite reactivity ( $k$ finite and $D \rightarrow 0$ ), we have $\tau_{1,1 d}^{\mathrm{opt}}=\sqrt{a / v k}(1 / 12 c)^{1 / 4}$ and the same optimal value equation (2) of $\tau_{2,1 d}^{\text {opt }}$. As in higher dimensions, $\tau_{2,1 d}^{\mathrm{opt}}$ depends neither on the thermal diffusion coefficient $D$ of phases 1 , nor on the association constant $k$, which shows that the optimal interaction time with motors $\tau_{2}^{\text {opt }}$ presents remarkable universal features. Furthermore, our approach permits an estimate of $\tau_{2}^{\text {opt }}$ compatible with observations in standard cellular conditions, which suggests that cellular transport could be close to optimum.

\section{METHODS}

The approximation scheme to solve the integro-differential equations (1) relies on the auxiliary function

$$
s(\mathbf{r})=\frac{1}{\Omega_{d}} \int t_{2} \mathrm{~d} \omega_{\mathbf{v}},
$$

and on the following decoupling hypothesis:

$$
\left\langle v_{i} v_{j} t_{2}\right\rangle_{\omega_{\mathbf{v}}} \simeq\left\langle v_{i} v_{j}\right\rangle_{\omega_{\mathbf{v}}}\left\langle t_{2}\right\rangle_{\omega_{\mathbf{v}}}=\frac{v^{2}}{d} \delta_{i j} s(\mathbf{r}) .
$$

Similar arguments as provided in ref. 23 then lead to the diffusion-like equation

$$
\widetilde{D} \Delta s(\mathbf{r})-\frac{1}{\tau_{2}}\left(s(\mathbf{r})-t_{1}\right)=-1,
$$

where $\widetilde{D}=v^{2} \tau_{2} / d$. After rewriting (1) as

$$
D \Delta t_{1}+\frac{1}{\tau_{1}}\left(s(\mathbf{r})-t_{1}\right)-k \mathrm{I}_{a}(\mathbf{r}) t_{1}=-1,
$$

equations (3) and (4) provide a closed system of linear differential equations for the variables $s$ and $t_{1}$, whose resolution is tedious but standard. For $d=3$, we obtain in the limit of perfect reaction $k \rightarrow \infty$ and low density $a \ll b$ :

$$
K_{3 d} \simeq \frac{3 \alpha_{1} \widetilde{D}\left(D \tau_{1} T\left(1+\alpha_{1} a\right)+\widetilde{D}\left(\tau_{1}+\tau_{2}\right)\left(\alpha_{2} a-T\right)\right)}{b^{3}\left(T+\alpha_{1} \alpha_{2} \widetilde{D}\left(\tau_{1}+\tau_{2}\right)\right)},
$$

where $\alpha_{1}=1 / \sqrt{D \tau_{1}}, \alpha_{2}=3 /\left(v \tau_{2}\right)$ and $T=\tanh \left(\alpha_{2} a\right)$. This decoupling assumption has been controlled numerically for a wide range of parameters for $d=2$ (ref. 23), and is shown here to also be satisfactory for $d=3$ (Fig. 2a,b). For $d=1$, the decoupling approximation is exact and yields after straightforward calculations an explicit though hardly handleable form of the reaction time. In the regime $D / v \ll a \ll b$, we obtain in the limit $k \rightarrow \infty$ the simple form

$$
K_{1 d}=\frac{a}{b} \frac{6 v s\left(a+\sqrt{D \tau_{1}}\right)}{\left(6 s v \tau_{1}+\sqrt{3 a b}\right)\left(a\left(1+4 s^{2}\right)+4 s^{2} \sqrt{D \tau_{1}}\right)},
$$

where $s=\left(\sqrt{a b} / v \tau_{2} \sqrt{3}\right)$. Optimization is then carried out using standard methods of functional analysis.
Received 1 September 2007; accepted 5 December 2007; published 13 January 2008.

\section{References}

1. Alberts, B. et al. Molecular Biology of the Cell (Garland, New York, 2002).

2. Thoumine, O. \& Ott, A. Time scale dependent viscoelastic and contractile regimes in fibroblasts probed by microplate manipulation. J. Cell. Sci. 110, 2109-2116 (1997).

3. Nedelec, F. J., Surrey, T., Maggs, A. C. \& Leibler, S. Self-organization of microtubules and motors. Nature 389, 305-308 (1997).

4. Le Goff, L., Amblard, F. \& Furst, E. M. Motor-driven dynamics in actin-myosin networks. Phys. Rev. Lett. 88, 018101 (2002).

5. Kruse, K., Joanny, J. F., Julicher, F., Prost, J. \& Sekimoto, K. Asters, vortices, and rotating spirals in active gels of polar filaments. Phys. Rev. Lett. 92, 078101 (2004).

6. Storm, C., Pastore, J. J., MacKintosh, F. C., Lubensky, T. C. \& Janmey, P. A. Nonlinear elasticity in biological gels. Nature 435, 191-194 (2005).

7. Voituriez, R., Joanny, J. F. \& Prost, J. Spontaneous flow transition in active polar gels. Europhys. Lett. 70, 404-410 (2005).

8. Voituriez, R., Joanny, J. F. \& Prost, J. Generic phase diagram of active polar films. Phys. Rev. Lett. 96 , $028102(2006)$.

9. Mizuno, D., Tardin, C., Schmidt, C. F. \& MacKintosh, F. C. Nonequilibrium mechanics of active cytoskeletal networks. Science 315, 370-373 (2007).

10. Dalhaimer, P., Discher, D. E. \& Lubensky, T. C. Crosslinked actin networks show liquid crystal elastomer behaviour, including soft-mode elasticity. Nature Phys. 3, 354-360 (2007).

11. Sheetz, M. P. \& Spudich, J. A. Movement of myosin-coated fluorescent beads on actin cables in vitro. Nature 303, 31-35 (1983).

12. Howard, J., Hudspeth, A. J. \& Vale, R. D. Movement of microtubules by single kinesin molecules. Nature 342, 154-158 (1989).

13. Ajdari, A. Transport by active filaments. Europhys. Lett. 31, 69-74 (1995).

14. Nedelec, F., Surrey, T. \& Maggs, A. C. Dynamic concentration of motors in microtubule arrays. Phys. Rev. Lett. 86, 3192 (2001).

15. Salman, H. et al. Nuclear localization signal peptides induce molecular delivery along microtubules. Biophys. J. 89, 2134-2145 (2005).

16. Huet, S., Karatekin, E., Tran, V. S., Cribier, S. \& Henry, J. P. Analysis of transient behavior in complex trajectories: Application to secretory vesicle dynamics. Biophys. J. 91, 3542-3559 (2006).

17. Shlesinger, M. F. \& Klafter, J. Random-walks in liquids. J. Phys. Chem. 93, 7023-7026 (1989).

18. Holcman, D. Modeling DNA and virus trafficking in the cell cytoplasm. J. Stat. Phys. 127, $471-494$ (2007).

19. Coppey, M., Bénichou, O., Voituriez, R. \& Moreau, M. Kinetics of target site localization of a protein on DNA: A stochastic approach. Biophys. J. 87, 1640-1649 (2004).

20. Slutsky, M. \& Mirny, L. A. Kinetics of protein-DNA interaction: Facilitated target location in sequence-dependent potential. Biophys. J. 87, 4021-4035 (2004).

21. Bénichou, O., Coppey, M., Moreau, M., Suet, P. H. \& Voituriez, R. Optimal search strategies for hidden targets. Phys. Rev. Lett. 94, 198101 (2005).

22. Lomholt, M. A., Ambjornsson, T. \& Metzler, R. Optimal target search on a fast-folding polymer chain with volume exchange. Phys. Rev. Lett. 95, 260603 (2005).

23. Bénichou, O., Loverdo, C., Moreau, M. \& Voituriez, R. Bidimensional intermittent search processes: an alternative to Lévy flights strategies. Phys. Rev. E 74, 020102 (2006).

24. Shlesinger, M. F. Mathematical physics: Search research. Nature 443, 281-282 (2006).

25. Bénichou, O., Loverdo, C., Moreau, M. \& Voituriez, R. A minimal model of intermittent search in dimension two. J. Phys. Condens. Matter 19, 065141 (2006).

26. Eliazar, I., Koren, T. \& Klafter, J. Searching circular DNA strands. J. Phys. Condens. Matter 19, 065140-065167 (2007)

27. Kolesov, G., Wunderlich, Z., Laikova, O. N., Gelfand, M. S. \& Mirny, L. A. How gene order is influenced by the biophysics of transcription regulation. Proc. Natl Acad. Sci. 104, 13948-13953 (2007).

28. Berg, O. \& Blomberg, C. Association kinetics with coupled diffusional flows. Special application to the lac repressor-operator system. Biophys. Chem. 4, 367-381 (1976).

29. Redner, S. A Guide to First- Passage Processes (Cambridge Univ. Press, Cambridge, 2001); errata: $<$ http://physics.bu.edu/ $/$ redner $/>$.

30. Condamin, S., Bénichou, O., Tejedor, V., Voituriez, R. \& Klafter, J. First-passage times in complex scale-invariant media. Nature 450, 77-80 (2007).

Correspondence and requests for materials should be addressed to R.V.

\section{Author contributions}

All authors contributed equally to this work.

Reprints and permission information is available online at http://npg.nature.com/reprintsandpermissions/ 